\title{
A new synthesis of novel alkenylated flavones by palladium- catalyzed cross-coupling reactions
}

\author{
Szabolcs Fekete, ${ }^{a}$ Tamás Patonay, ${ }^{\text {** }}$ Artur M. S. Silva, ${ }^{\text {b }}$ and José A. S. Cavaleiro ${ }^{b}$ \\ ${ }^{a}$ Department of Organic Chemistry, University of Debrecen, H-4010 Debrecen, \\ P.O.B. 20, Hungary \\ ${ }^{b}$ Department of Chemistry \& QOPNA, University of Aveiro, 3810-193 Aveiro, Portugal \\ E-mail: patonay.tamas@science.unideb.hu
}

\section{Dedicated to Prof. Ferenc Fülöp on the occasion of his $60^{\text {th }}$ birthday}

\begin{abstract}
Bromoflavones were treated with various terminal alkynes in palladium-catalyzed cross-coupling reactions under phosphine-free condions to give the expected alkenylated flavones in moderate to good yields. The presence of two different $\beta$ hydrogens in the terminal alkene led to the formation of both alkenylated and alkylated products.
\end{abstract}

Keywords: Alkenylation, C-C bond formation, flavone, Heck reaction, Jeffery's conditions

\section{Introduction}

Flavones are relatively common natural products in the Plant Kingdom. They play important roles in the plant reproduction and defense and also possess a wide range of biological and pharmaceutical activities. ${ }^{1}$ Both natural and synthetic derivatives show marked antiproliferative, anticarcinogenic or antitumor effect ${ }^{2}$ and, among others, antioxidant, ${ }^{3}$ anticonvulsive, ${ }^{4} \alpha-$ glucosidase inhibitor, ${ }^{5}$ aldehyde dehydrogenase ALDH-2 inhibitor, ${ }^{6}$ EGFR tyrosine kinase ${ }^{7}$ and COX-2 inhibiting activity. ${ }^{8}$

The group of 2- and 3-styrylchromones is a smaller family of chromonoids ${ }^{9,10}$ but few representatives are known in the nature and their biological activities were also reported and reviewed. ${ }^{11}$ Much less has been reported about 3-styrylflavones. ${ }^{12}$ The first contribution is related to Parthasarathy's group in the early 90 's, ${ }^{12 a}$ they have synthetized the target 3 styrylflavone from 3-methylflavone by using Wittig reaction. Another synthetic approach based on the condensation of 1-(2-hydroxyphenyl)-3-phenylpropane-1,3-diones and 
phenylacetaldehydes. ${ }^{12 \mathrm{~b}}$ An interesting synthesis of 3-vinyl- or 3-(1-propenyl)flavones and related systems by using an acid-catalyzed retro-Michael ring opening of 3-acylchromanones was also published. ${ }^{13}$

An alternative approach to alkenylchromones and flavones is the palladium-catalyzed crosscoupling reaction (Heck reaction) of halochromones or -flavones with various alkenes. Surprisingly, only sporadic contributions were presented till the end of the last decade. ${ }^{14}$ Recently, we presented the results of our detailed studies on the Heck reaction of various bromochromones and their derivatives with a protected ortho-hydroxy group. It was proved that this effective reaction offers a new entry to naturally occurring derivatives. ${ }^{15}$ Presence of the ortho-hydroxy functionality allows a ring-closure to give tricyclic systems. In this contribution we wish to display some of our results in the field of cross-coupling of bromoflavones. Obviously, this new alkenylation methodology opens up a new way to a wide group of natural products, some representative examples are shown in Figure 1.<smiles>COc1ccc(-c2cc(=O)c3c(O)c(/C=C/C(=O)O)c(O)cc3o2)cc1O</smiles>

Torosaflavone D [16]<smiles>CC1(C)C=Cc2c(cc3oc(-c4ccc(O)cc4)cc(=O)c3c2O)O1</smiles>

Carpachromene [19]<smiles>CC(=O)C=Cc1c(O)cc2oc(-c3ccc(O)cc3)cc(=O)c2c1O</smiles>

Anadanthoflavone [17]<smiles>CC1(C)C=Cc2c(cc(O)c3c(=O)cc(-c4ccc(O)cc4)oc23)O1</smiles>

Atalantoflavone [20]<smiles>COc1c(-c2ccccc2)oc2c(ccc3occc32)c1=O</smiles>

Karanjin [21]

\section{Figure 1}

Several flavones or isoflavones with an alkenyl side-chain or 2-arylfuro- or -pyranochromone structure show biological activity. For instance, anadanthoflavone is active against both human platelet 12-lipoxygenase and human reticulocyte 15 -lipoxygenase, ${ }^{17}$ isoartocarpesin inhibits tyrosinase and, thus, melanin biosynthesis. ${ }^{18}$ Carpachromene had considerable cytotoxic effect against various cell lines. ${ }^{19 \mathrm{~b}}$ Atalantoflavone was found to be active against the plant pathogen Xylella fastidiosa. ${ }^{20 \mathrm{~b}}$ Karanjin and related derivatives show antihyperglycemic ( $\alpha$-glucosidase inhibitor) effect. ${ }^{21 \mathrm{~b}}$ 


\section{Results and Discussion}

Alkenylation of flavone ring system at position 3 was tested first. The starting material 3bromoflavones 4-6 were prepared by an N-bromosuccinimide (NBS) bromination of the corresponding flavones 1-3 in the presence of substoichiometric amount of pyridine, ${ }^{22}$ the yields varied in wide range depending on the substituent in position 4'. Our previous studies in the field of bromochromones showed the high efficiency of the phoshine-free conditions using quaternary ammonium salts frequently cited as Jeffery's conditions. ${ }^{15}$ To our delight this method (Conditions A) worked well in the case of 3-bromoflavones 4-6, too (Scheme 1, Table 1). The reactions of 3-bromoflavones $\mathbf{4 , 5}$ with styrenes $\mathbf{7 a - d}$ gave the expected 3-styrylflavones 8a-d, 9a,b in moderate to good (51-95\%) yields (Table 1, Entries 1-4, 8,9). The reactivity of 3-bromo4 '-chloroflavone (6), however, was considerably lower, a poor $(45 \%)$ yield was found even at elevated temperature and extended reaction period (Table 1, Entry 11). Other terminal alkenes such as 2-methylbut-3-en-2-ol (7e) or ethyl acrylate (7f) led also to the desired products 8e,f, 9f and $10 f$ but a higher temperature and longer reaction period was needed for the completion of the reaction in the case of ethyl acrylate (7f) (Table 1, Entries 6,10,14). In the reaction of 3bromochromone (4) and ethyl acrylate (7f) we have also tested the phosphine-containing conditions (Conditions B, Scheme 1) but no improvement of the yield was observed even at 155 ${ }^{\circ} \mathrm{C}$ (Table 1, Entry 7). The low reactivity of substrate 6 was found, again. Its reaction with butenol 7e failed to give the expected product 10e under Conditions A or B (Table 1, Entries 12,13). The reason of the decreased reactivity is not clear, the electron-withdrawing chlorine should stabilize the organopalladium intermediate and increase the rate.

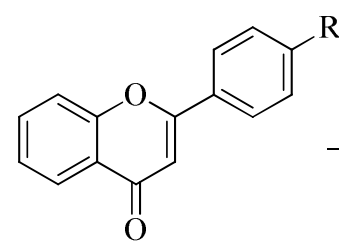

1-3

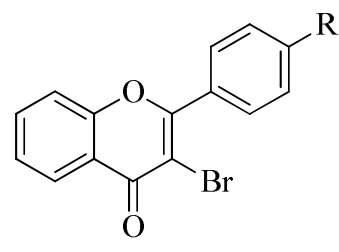

4-6

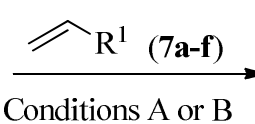

Conditions A or B

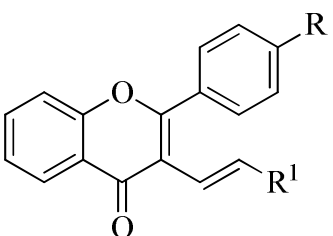

8-10

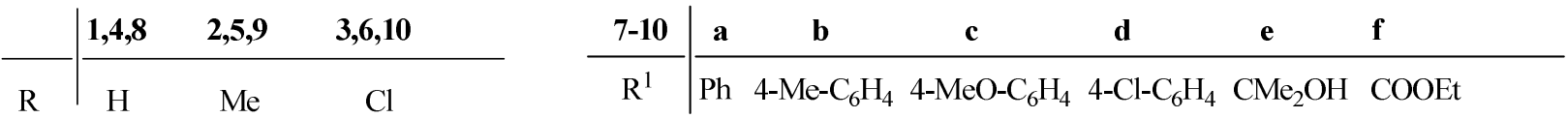

Scheme 1. Conditions $\mathrm{A}: \mathrm{Pd}(\mathrm{OAc})_{2}, \mathrm{~K}_{2} \mathrm{CO}_{3}$, tetrabutylammonium bromide (TBAB), $\mathrm{KCl}$, $\mathrm{DMF} /$ heating. Conditions $\mathrm{B}: \mathrm{Pd}(\mathrm{OAc})_{2}$, triethyl amine (TEA), triphenyl phosphine (TPP), NMP/heating. 
Table 1. Reaction of 3-bromoflavones 4-6 with various terminal alkenes 7a-f

\begin{tabular}{ccccccc}
\hline Entry & Substrate & Product & Conditions & $\begin{array}{c}\text { Temperature } \\
\left({ }^{\circ} \mathrm{C}\right)\end{array}$ & $\begin{array}{c}\text { Reaction period } \\
(\min )\end{array}$ & $\begin{array}{c}\text { Yield } \\
(\%)\end{array}$ \\
\hline 1 & $\mathbf{4}$ & $\mathbf{8 a}$ & A & 100 & 35 & 76 \\
2 & $\mathbf{4}$ & $\mathbf{8 b}$ & A & 110 & 30 & 95 \\
3 & $\mathbf{4}$ & $\mathbf{8 c}$ & A & 110 & 30 & 77 \\
4 & $\mathbf{4}$ & $\mathbf{8 d}$ & A & 110 & 40 & 94 \\
5 & $\mathbf{4}$ & $\mathbf{8 e}$ & A & 110 & 25 & 81 \\
6 & $\mathbf{4}$ & $\mathbf{8 f}$ & A & 140 & 240 & 65 \\
7 & $\mathbf{4}$ & $\mathbf{8 f}$ & B & 155 & 150 & 69 \\
8 & $\mathbf{5}$ & $\mathbf{9 a}$ & A & 110 & 50 & 51 \\
9 & $\mathbf{5}$ & $\mathbf{9 b}$ & A & 110 & 45 & 94 \\
10 & $\mathbf{5}$ & $\mathbf{9 f}$ & A & 140 & 60 & 76 \\
11 & $\mathbf{6}$ & $\mathbf{1 0 a}$ & A & 140 & 540 & 45 \\
12 & $\mathbf{6}$ & $\mathbf{1 0 e}$ & A & 140 & 3 days & traces \\
13 & $\mathbf{6}$ & $\mathbf{1 0 e}$ & B & 140 & $20 \mathrm{~h}$ & traces \\
14 & $\mathbf{6}$ & $\mathbf{1 0 f}$ & A & 140 & 40 & 45 \\
\hline
\end{tabular}

As usual, the cross-coupling reactions took place with complete diastereoselectivity, only the thermodynamically more stable $E$ alkenes were obtained. The relative configuration was proven by the high (ca. $16 \mathrm{~Hz}$ ) value of ${ }^{3} J_{\mathrm{H}, \mathrm{H}}$ coupling constants of the vinylic hydrogens. Most of these products were hitherto unknown in the literature.

We also investigated the Heck reaction of 6-bromo- (15) and 7-bromoflavone (16). The necessary starting materials $\mathbf{1 3 , 1 4}$ were prepared by our oxidative cyclization methodology ${ }^{23}$ and their reaction with the substituted styrenes 7a-d and 2-methylbut-3-en-2-ol (7e) gave the corresponding $E$-alkenes $\mathbf{1 5 , 1 6}$ smoothly and usually in good yields (Scheme 2, Table 2).

It is noteworthy that ethyl acrylate (7f) reacted more sluggishly, again. Only reactions under Conditions $\mathrm{B}$ at higher $\left(160{ }^{\circ} \mathrm{C}\right)$ temperature and long reaction periods afforded the desired acrylates $15 f$ and 16f. To our best knowledge, this is the first reported synthesis flavones having 1 -alkenyl groups in their Ring A.

In addition, we studied the cross-coupling of 6-bromoflavone (13) with acrolein diethyl acetal (17) and, in accordance with our observation made in the bromochromone series, ${ }^{15 a}$ two products were isolated. The major one was ethyl (E)-6-(2-phenyl-4-oxo-4H-1-benzopyran-3yl)propionate (19) while the minor one was identified as the expected acrylaldehyde (18). The reason of this double reactivity is the presence of two different $\beta$ hydrogens in contrast with the previous terminal alkenes having only one $\beta$ hydrogen. The competitive $\beta$ and $\beta$ ' hydride eliminations in the catalytic cycle gave these two products. ${ }^{14 a, 15}$ This double reactivity pattern seems to be characteristic for Jeffery's conditions and allows the synthesis of flavonylpropionates by Heck reaction. 
<smiles></smiles>

11,12

13,14

15,16

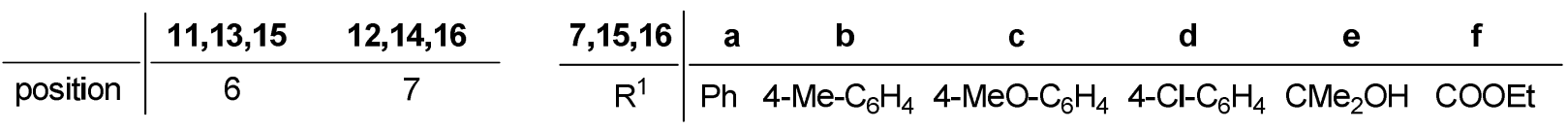<smiles>C=CC(OCC)OCC</smiles>

13

18

19

Scheme 2. Conditions A: $\mathrm{Pd}(\mathrm{OAc})_{2}, \mathrm{~K}_{2} \mathrm{CO}_{3}$, tetrabutylammonium bromide (TBAB), $\mathrm{KCl}$, $\mathrm{DMF} /$ heating. Conditions $\mathrm{B}: \mathrm{Pd}(\mathrm{OAc})_{2}$, triethyl amine (TEA), triphenyl phosphine (TPP), NMP/heating.

Table 2. Reaction of 6-bromoflavone (13) and 7-bromoflavone (14) with various terminal alkenes $7 \mathbf{a}-\mathbf{f}$

\begin{tabular}{ccccccc}
\hline Entry & Substrate & Product & Conditions & $\begin{array}{c}\text { Temperature } \\
\left({ }^{\circ} \mathrm{C}\right)\end{array}$ & $\begin{array}{c}\text { Reaction period } \\
(\mathrm{min})\end{array}$ & $\begin{array}{c}\text { Yield } \\
(\%)\end{array}$ \\
\hline 1 & $\mathbf{1 3}$ & $\mathbf{1 5 a}$ & A & 110 & 60 & 87 \\
2 & $\mathbf{1 3}$ & $\mathbf{1 5 b}$ & A & 110 & 40 & 88 \\
3 & $\mathbf{1 3}$ & $\mathbf{1 5 c}$ & A & 110 & 30 & 41 \\
4 & $\mathbf{1 3}$ & $\mathbf{1 5 d}$ & A & 110 & 30 & 87 \\
5 & $\mathbf{1 3}$ & $\mathbf{1 5 e}$ & A & 110 & 30 & 77 \\
6 & $\mathbf{1 3}$ & $\mathbf{1 5 f}$ & B & 160 & 300 & 32 \\
7 & $\mathbf{1 4}$ & $\mathbf{1 6 a}$ & A & 110 & 60 & 65 \\
8 & $\mathbf{1 4}$ & $\mathbf{1 6 b}$ & A & 110 & 60 & 92 \\
9 & $\mathbf{1 4}$ & $\mathbf{1 6 c}$ & A & 110 & 40 & 89 \\
10 & $\mathbf{1 4}$ & $\mathbf{1 6 d}$ & A & 110 & 90 & 68 \\
11 & $\mathbf{1 4}$ & $\mathbf{1 6 e}$ & A & 110 & 40 & 76 \\
12 & $\mathbf{1 4}$ & $\mathbf{1 6 f}$ & B & 160 & 150 & 84 \\
\hline
\end{tabular}




\section{Conclusions}

In conclusion, our previous synthetic route developed for the synthesis of alkenylated chromones was successfully extended to flavones as we demonstrated that Heck reaction of various bromoflavones under modified Jeffery's conditions resulted in a wide range of hitherto unknown alkenylated flavones in good yields. This efficient methodology opens up the way not only to flavonyl alkenes but also to naturally occurring flavone derivatives with resacetophenone or phloroacetophenone-type substitution pattern. The competitive $\beta$ and $\beta$ ' hydride eliminations offers a further synthetic tool for the preparation of flavones with a new $\mathrm{C}(\mathrm{Ar}) \mathrm{C}(\mathrm{alkyl})$ bond.

\section{Experimental Section}

General. Chromatographic separations were performed using silica gel (Merck, 70-230 mesh). Thin-layer chromatography was carried out on Kieselgel $60 \mathrm{~F}_{254}(0.25 \mathrm{~mm}$ layer thickness, Merck). Melting points were determined on a Boetius hot-stage apparatus and are uncorrected. ${ }^{1} \mathrm{H}$ NMR and ${ }^{13} \mathrm{C}$ NMR spectra were recorded with a Bruker AM $360\left(360 \mathrm{MHz}\right.$ for ${ }^{1} \mathrm{H} ; 90 \mathrm{MHz}$ for ${ }^{13} \mathrm{C}$ nuclei) spectrometer in $\mathrm{CDCl}_{3}$ solution unless otherwise specified (internal standard TMS,$\delta=0 \mathrm{ppm}$ or residual peaks $\mathrm{CHCl}_{3}(\delta=7.26 \mathrm{ppm})$ for ${ }^{1} \mathrm{H} \mathrm{NMR}$ and $(\delta=77.00)$ for ${ }^{13} \mathrm{C}$ NMR). ${ }^{13} \mathrm{C}$ NMR assignments were supported by J-echo technique. IR spectra were recorded with a Perkin-Elmer 16 PC-FT-IR or Jasco FT-IR 4100A instrument in KBr disks. Elemental analyses were performed in house with an Elementar Vario MicroCube instrument.

Bromoflavones. 3-Bromoflavones 4-6 were prepared according to a literature procedure ${ }^{22}$ by heating the corresponding flavones 1a-b with 1.67 equiv. of N-bromosuccinimide (NBS) and 0.25 equiv. of pyridine in dry carbon tetrachloride $(12 \mathrm{~mL})$. 3-Bromoflavone $(4)^{24}$ and 3bromoflavones $\mathbf{5 , 6}{ }^{25}$ were identified by their literature mp values and NMR spectra. Yields: $77 \%$ (4), 26\% (5), 44\% (6). 6-Bromoflavone (13) and 7-bromoflavone (14) were synthetized by our methodology (iodine-catalyzed dehydrogenation in hot DMSO solution). ${ }^{23}$ Bromoflavones $\mathbf{1 3}^{26}$ and $14^{27}$ were identified by their literature mp values and NMR characteristics. Yields: $53 \%$ (13), $66 \%(14)$.

\section{General procedure for the synthesis of alkenylated flavones}

Conditions A. A mixture of bromoflavone 4-6, 11,13 (1.25 mmol), potassium carbonate (1.87 $\mathrm{mmol})$, potassium chloride $(1.25 \mathrm{mmol})$, TBAB $(2.52 \mathrm{mmol})$, palladium(II) acetate $(0.08 \mathrm{mmol})$ and alkene 7a-f $(4.16 \mathrm{mmol})$ in DMF $(10 \mathrm{~mL})$ was heated at the temperature given in Tables 1 and 2 in an oil bath under nitrogen atmosphere. After the completion of reaction (TLC monitoring, hexane-ethyl acetate $=1: 1, \mathrm{v} / \mathrm{v}$ ) the mixture was poured into water, the precipitation was filtered off and purified by recrystallization or by column chromatography. When the product did not precipitate the mixture was extracted with diethyl ether (3x35 mL), the combined organic layers were washed with water, dried $\left(\mathrm{MgSO}_{4}\right)$ and concentrated under reduced pressure. 
Conditions B. A mixture of bromoflavone 4-6, 11,13 (1.25 mmol), TEA (1.39 mmol), TPP (0.12 $\mathrm{mmol})$, palladium(II) acetate $(0.008 \mathrm{mmol})$ and alkene $\mathbf{7 a - f}(4.16 \mathrm{mmol})$ in NMP $(10 \mathrm{~mL})$ was heated (see Tables 1 and 2) in an oil bath under nitrogen atmosphere. After the completion of reaction (TLC monitoring, hexane-ethyl acetate $=1: 1, \mathrm{v} / \mathrm{v}$ ) the mixture was worked up as stated above.

(E)-3-Styrylflavone (8a). Method A, reaction period: $35 \mathrm{~min}$. Recrystallization from hexaneethyl acetate. Yield: $76 \%$. Brownish crystalline powder, mp 173-174 ${ }^{\circ} \mathrm{C}$. IR: $v_{\max } 3061,1641$ $(\mathrm{C}=\mathrm{O}), 1614(\mathrm{C}=\mathrm{C}), 1545,1467,1390$ (flavone skeleton), 1231, $964(\mathrm{CH}=\mathrm{CH}$, trans), 769, 754, $700 \mathrm{~cm}^{-1}$; ${ }^{1} \mathrm{H}$ NMR: $\delta 6.83(\mathrm{~d}, J=16.1 \mathrm{~Hz}, 1 \mathrm{H}, \beta-\mathrm{H}), 7.25-7.75(\mathrm{~m}, 14 \mathrm{H}, \mathrm{Ar}-\mathrm{H}), 8.02(\mathrm{~d}, J=$ $16.1 \mathrm{~Hz}, 1 \mathrm{H}, \alpha-\mathrm{H}), 8.31(\mathrm{~d}, J=7.6 \mathrm{~Hz}, 1 \mathrm{H}, 5-\mathrm{H}) ;{ }^{13} \mathrm{C}-\mathrm{NMR}: \delta 117.5(\mathrm{C}-3), 117.8(\mathrm{C}-8), 120.0$

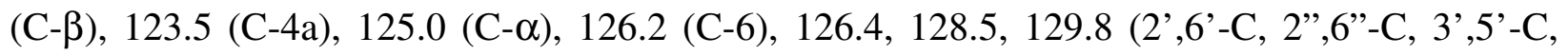
3",5”-C), 127.5 (C-4”), 130.7 (C-4'), 133.1 (C-1'), 134.3 (C-5), 138.1 (C-7), 155.3 (C-1”), 163.0 (C-8a), 165.8 (C-2), 177.4 (C-4). Anal. Calcd. for $\mathrm{C}_{23} \mathrm{H}_{16} \mathrm{O}_{2}$ (324.38): C, 85.16; H, 4.97\%. Found: C, 84.98; H, 5.11\%.

(E)-3-(4-Methylstyryl)flavone $(\mathbf{8 b})$. Method A, reaction period: $30 \mathrm{~min}$. Purified by column chromatography (eluent: hexane-ethyl acetate $=1: 1$, v/v). Yield: 95\%. Yellow powder, mp 160$161{ }^{\circ} \mathrm{C}$. IR: $v_{\max } 3016,1639(\mathrm{C}=\mathrm{O}), 1614(\mathrm{C}=\mathrm{C}), 1547,1464,1396$ (flavone skeleton), 1227, 1176, 1115, $967(\mathrm{HC}=\mathrm{CH}$, trans $), 772,755,704 \mathrm{~cm}^{-1} ;{ }^{1} \mathrm{H} \mathrm{NMR}: \delta 2.31\left(\mathrm{~s}, 3 \mathrm{H}, \mathrm{CH}_{3}\right), 6.75(\mathrm{~d}, J=$ $16.1 \mathrm{~Hz}, 1 \mathrm{H}, \beta-\mathrm{H}), 7.08(\mathrm{~d}, J=7.7 \mathrm{~Hz}, 2 \mathrm{H}, 3$, 5"-H), 7.26 (d, $J=7.7 \mathrm{~Hz}, 2 \mathrm{H}, 2$ 2",6"-H), 7.387.53 (m, 5H, 6-H, 8-H, 3',5'-H, 4'-H), 7.63 (m, 1H, 7-H), 7.73 (m, 2H, 2',6'-H), 7.95 (d, J = $16.1 \mathrm{~Hz}, 1 \mathrm{H}, \alpha-\mathrm{H}) ; 8.30(\mathrm{~d}, J=7.7 \mathrm{~Hz}, 1 \mathrm{H}, 5-\mathrm{H}) ;{ }^{13} \mathrm{C}-\mathrm{NMR}: \delta 21.1\left(\mathrm{CH}_{3}\right), 117.7(\mathrm{C}-8), 117.8$ (C-3), 119.0 (C- $\beta$ ), 123.5 (C-4a), 124.9, 126.2 (C-6, C-4'), 126.3 (C-3',5'), 128.4, 129.2, 129.7 (C-2',6', C-2",6”, C-3",5”), 130.7 (C- $\alpha$ ), 133.2 (C-1'), 133.3 (C-5), 134.3 (C-7), 135.3 (C-1”), 137.3 (C-4”), 155.3 (C-8a), 165.2 (C-2), 177.4 (C-4). Anal. Calcd. for $\mathrm{C}_{24} \mathrm{H}_{18} \mathrm{O}_{2}$ (338.41): C, 85.18; H, 5.36\%. Found: C, 85.29; H, 5.44\%.

(E)-3-(4-Methoxystyryl)flavone $(\mathbf{8 c})$. Method A, reaction period: $30 \mathrm{~min}$. Recrystallization from hexane - abs. ethanol. Yield: $77 \%$. Brownish crystalline powder, mp 114-116 ${ }^{\circ} \mathrm{C}$. IR: $v_{\max }$ 3077, 2960, $2833\left(\mathrm{CH}_{3} \mathrm{O}\right), 1636(\mathrm{C}=\mathrm{O}), 1613(\mathrm{C}=\mathrm{C}), 1509,1443,1390$ (flavone skeleton), 1304, 1278, 1245 (C-O-C), 1181, 1172, 1110, 1036 (C-O-C), 970 (HC=CH, trans), 820, 777, 762, 704 $\mathrm{cm}^{-1}$; ${ }^{1} \mathrm{H}$ NMR: $\delta 3.79\left(\mathrm{~s}, 3 \mathrm{H}, \mathrm{CH}_{3} \mathrm{O}\right), 6.61(\mathrm{~d}, J=16.8 \mathrm{~Hz}, 1 \mathrm{H}, \beta-\mathrm{H}), 6.82(\mathrm{~d}, J=9.1 \mathrm{~Hz}, 2 \mathrm{H}$, 3",5"-H); 7.30 (d, J = 9.1 Hz, 2H, 2",6"-H); 7.32-7.48 (m, 2H, 6-H, 8-H), 7.58-7.60 (m, 3H, 3',5'-H, 4'-H), 7.63 (m, 1H, 7-H), 7.72 (m, 2H, 2',6'-H); 7.94 (d, J = 16.8 Hz, 1H, $\alpha-\mathrm{H}), 8.30$ (d, $J=8.4 \mathrm{~Hz}, 1 \mathrm{H}, 5-\mathrm{H}) ;{ }^{13} \mathrm{C}-\mathrm{NMR}: \delta 55.2\left(\mathrm{CH}_{3} \mathrm{O}\right), 113.9(\mathrm{C}-3 ", 5 ”), 117.7(\mathrm{C}-3), 117.8,117.9(\mathrm{C}-8$, C-ß), 123.4 (C-4a), 124.9 (C-6), 126.2 (C-4'), 127.6, 128.4, 129.7 (C-2',6', C-3',5', C-2”,6”); 130.5 (C- $\alpha$ ), 130.9 (C-1',1”), 133.3, 133.9 (C-5, C-7), 155.3 (C-8a), 159.2 (C-4”), 162.5 (C-2), 177.5 (C-4). Anal. Calcd. for $\mathrm{C}_{24} \mathrm{H}_{18} \mathrm{O}_{3}$ (354.41): C, 81.34, H, 5.12\%. Found: C, 81.50; H, $5.07 \%$.

(E)-3-(4-Chlorostyryl)flavone $(\mathbf{8 d})$. Method A, reaction period: $40 \mathrm{~min}$. Purified by column chromatography (eluent: hexane-ethyl acetate $=1: 1$, v/v). Yield: 94\%. Yellow crystalline powder, mp 146-148 ${ }^{\circ} \mathrm{C}$. IR: $v_{\max } 3058,1640(\mathrm{C}=\mathrm{O}), 1611(\mathrm{C}=\mathrm{C}), 1543,1491,1465,1445,1395$ 
(flavone skeleton), 1336, 1312, 1292, 1228, 1118, 1095, 1078, $968(\mathrm{HC}=\mathrm{CH}$, trans $), 915$, 821,769, 757, $700 \mathrm{~cm}^{-1}$; ${ }^{1} \mathrm{H}$ NMR: $\delta 6.75(\mathrm{~d}, J=16.1 \mathrm{~Hz}, 1 \mathrm{H}, \beta-\mathrm{H}), 6.79-7.29(\mathrm{~m}, 4 \mathrm{H}, 2 ", 3 ”-\mathrm{H}$, 5",6"'-H), 7.40-7.48 (m, 2H, 6, 8-H), 7.55 (m, 3H, 3', 5'-H, 4'-H), 7.64 (m, 1H, 7-H), 7.71 (m, 2H, 2',6'-H), $7.97(\mathrm{~d}, J=16.1 \mathrm{~Hz}, 1 \mathrm{H}, \alpha-\mathrm{H}), 8.29(\mathrm{~d}, J=8.3 \mathrm{~Hz}, 1 \mathrm{H}, 5-\mathrm{H}) ;{ }^{13} \mathrm{C}-\mathrm{NMR}: \delta 117.2$ (C-3), 117.8 (C-8), 120.7 (C- $\beta$ ), 123.4 (C-4a), 125.1 (C-6), 126.2 (C-4'), 127.5, 128.5, 128.6, 129.7 (C-2',6', C-3',5', C-2”,6”, C-3",5”), 130.8 (C-8), 132.9, 133.5 (C-5, C- $\alpha$ ), 133.0 (C-1', C4"), 136.6 (C-1"), 155.3 (C-8a), 163.2 (C-2), 177.3 (4-C). Anal. Calcd. for $\mathrm{C}_{23} \mathrm{H}_{15} \mathrm{ClO}_{2}$ (358.82): C, 76.99; H, 4.21\%. Found: C, 80.11; H, 4.39\%.

(E)-3-(3-Hidroxy-3-methylbut-1-enyl)flavone (8e). Method A, reaction period: $25 \mathrm{~min}$. Purified by column chromatography (eluent: toluene-ethyl acetate $=4: 1, \mathrm{v} / \mathrm{v}$ ). Yield: $81 \%$. Yellow crystalline powder, mp 103-104 ${ }^{\circ} \mathrm{C}$. IR: $v_{\max } 3420(\mathrm{OH}), 2975,1640(\mathrm{C}=\mathrm{O}), 1619(\mathrm{C}=\mathrm{C})$, 1543, 1466, 1399 (flavone skeleton), 1372, 1268 (C-O), 1235, 1203, 1157, 1121, 985 (CH=CH, trans), 916, 783, 758, $708 \mathrm{~cm}^{-1}$; ${ }^{1} \mathrm{H}$ NMR: $\delta 1.33\left(\mathrm{~s}, 6 \mathrm{H}, 2 \mathrm{xCH}_{3}\right), 1.68(\mathrm{~s}, 1 \mathrm{H}, \mathrm{OH}), 6.38(\mathrm{~d}, J=$ $16.1 \mathrm{~Hz}, 1 \mathrm{H}, 2$ '-H), 7.01 (d, $J=16.1 \mathrm{~Hz}, 1 \mathrm{H}, 1$ '-H), 7.40-7.52 (m, 5H, 6-H, 8-H, 3',5'-H, 4'-H), 7.64-7.69 (m, 3H, 7-H, 2',6'-H), $8.26(\mathrm{~d}, J=7.7 \mathrm{~Hz}, 1 \mathrm{H}, 5-\mathrm{H}) ;{ }^{13} \mathrm{C}-\mathrm{NMR}: \delta 29.6\left(2 \mathrm{xCH}_{3}\right), 71.2$ (C-3"), 117.3, 117.7 (C-8, C-1"), 117.5 (C-3), 123.3 (C-4a), 125.0, 126.1 (C-6, C-4'), 128.3 (C3',5'), 129.6 (C-2',6'), 130.5 (C-2'), 133.1 (C-1'), 133.4 (C-5), 143.9 (C-7), 155.4 (C-8a), 162.5 (C-2), 177.5 (C-4). Anal. Calcd. for $\mathrm{C}_{20} \mathrm{H}_{18} \mathrm{O}_{3}$ (306.36): C, 78.41; H, 5.92\%. Found: C, 78.35; H, $5.85 \%$.

Ethyl (E)-3-(2-phenyl-4-oxo-4H-1-benzopyran-3-yl)acrylate (8f). Method A, reaction period: 240 min. Recrystallization from hexane - abs. ethanol. Yield: 65\%. Method B, reaction period: $155 \mathrm{~min}$. Recrystallization from diisopropyl ether. Yield: $69 \%$. Brownish crystalline powder, $\mathrm{mp}$ 153-154 ${ }^{\circ} \mathrm{C}$. IR: $v_{\max } 2976\left(\mathrm{CH}_{3}\right), 1711(\mathrm{C}=\mathrm{O}$, ester $), 1653(\mathrm{C}=\mathrm{O}$, ketone), $1616(\mathrm{C}=\mathrm{C}), 1467$, 1400 (flavone skeleton), 1297, 1276 (C-O-C), 754, $689 \mathrm{~cm}^{-1} ;{ }^{1} \mathrm{H}$ NMR: $\delta 1.26(\mathrm{t}, J=7.0 \mathrm{~Hz}, 3 \mathrm{H}$, $\mathbf{C H}_{3} \mathrm{CH}_{2}$ ), 4.17 (q, $J=7.0 \mathrm{~Hz}, 2 \mathrm{H}, \mathrm{CH}_{3} \mathbf{C H}_{2}$ ), 7.42-7.50 (m, 4H, 6'-H, 8'-H, 4'-H, 2-H), 7.527.59 (m, 3H, 7-H, 3",5"-H), 7.65-7.72 (m, 3H, 2",6"-H, 3-H), 8.28 (d, $J=7.7 \mathrm{~Hz}, 1 \mathrm{H}, 5$ '-H); ${ }^{13}$ C-NMR: $\left.\delta 14.2 \mathbf{C H}_{3} \mathrm{CH}_{2}\right), 60.2\left(\mathrm{CH}_{3} \mathbf{C H}_{2}\right), 115.4\left(\mathrm{C}-3\right.$ ') $117.8\left(\mathrm{C}-8^{\prime}\right), 123.3(\mathrm{C}-2), 125.5$, 126.3 (C-6', C-4”), 128.6 (C-3",5”), 129.8 (C-2",6”), 131.4 (C-5'), 132.1 (C-1"), 133.8 (C-7'), 135.9 (C-3), 155.2 (C-8a'), 166.7, 167.8 (C-2', C-1), 176.8 (C-4'). Anal. Calcd. for $\mathrm{C}_{20} \mathrm{H}_{16} \mathrm{O}_{4}$ (320.34): C, 74.99; H, 5.03\%. Found: C, 74.79; H, 5.16\%.

(E)-4'-Methyl-3-styrylflavone (9a). Method A, reaction period: $50 \mathrm{~min}$. Recrystallization from diisopropyl ether. Yield: $51 \%$. Light yellow crystalline powder, mp $124-125{ }^{\circ} \mathrm{C}$. IR: $v_{\max } 3019$, $1640(\mathrm{C}=\mathrm{O}), 1613(\mathrm{C}=\mathrm{C}), 1549,1467,1387$ (flavone skeleton), 1228, 1186, 1106, $966(\mathrm{CH}=\mathrm{CH}$, trans), 817, 755, $699 \mathrm{~cm}^{-1}$; ${ }^{1} \mathrm{H}$ NMR: $\delta 2.48\left(\mathrm{~s}, 3 \mathrm{H}, \mathrm{CH}_{3}\right), 6.84(\mathrm{~d}, J=16.1 \mathrm{~Hz}, 1 \mathrm{H}, \beta-\mathrm{H}), 7.20-$ 7.49 (m, 9H, Ar-H), 7.64-7.69 (m, 3H, 7-H, 2',6'-H), 8.04 (d, $J=16.1 \mathrm{~Hz}, 1 \mathrm{H}, \alpha-\mathrm{H}), 8.31$ (d, $J=$ $7.2 \mathrm{~Hz}, 1 \mathrm{H}, 5-\mathrm{H}) ;{ }^{13} \mathrm{C}-\mathrm{NMR}: \delta 21.5\left(\mathrm{CH}_{3}\right), 117.2(\mathrm{C}-3), 117.7(\mathrm{C}-8), 120.3(\mathrm{C}-\beta), 123.5(\mathrm{C}-4 \mathrm{a})$, 124.9, 126.1, 127.3 (C-6, C-4", C- $\alpha$ ), 126.4 (C-3",5”), 128.5, 129.1, 129.7 (C-2',6', C-3',5', C2",6”), 130.2 (C-1'), 133.3, 134.0 (C-5, C-7), 138.2 (C-1"), 141.1 (C-4'), 155.3 (C-8a), 163.2 (C2), 177.4 (C-4). Anal. Calcd. for $\mathrm{C}_{24} \mathrm{H}_{18} \mathrm{O}_{2}$ (338.41): C, 85.18; H, 5.36\%. Found: C, 85.07; H, $5.35 \%$. 
(E)-3-(3-Hidroxy-3-methylbut-1-enyl)-4'-methylflavone (9e). Method A, reaction period: 45 min. Purified by column chromatography (eluent: toluene-ethyl acetate $=4: 1$, v/v). Yield: $94 \%$. Light yellow crystalline powder, mp 75-76 ${ }^{\circ} \mathrm{C}$. IR: $v_{\max } 3426(\mathrm{OH}), 2973,1643(\mathrm{C}=\mathrm{O}), 1613$ $(\mathrm{C}=\mathrm{C}), 1545,1466,1394$ (flavone skeleton), $1266(\mathrm{C}-\mathrm{OH}), 1234,1110,918,758 \mathrm{~cm}^{-1}$; ${ }^{1} \mathrm{H}$ NMR: $\delta 1.35\left(\mathrm{~s}, 6 \mathrm{H}, 2 \mathrm{xCH}_{3}\right), 2.35(\mathrm{~s}, 1 \mathrm{H}, \mathrm{OH}), 2.44\left(\mathrm{~s}, 3 \mathrm{H}, 4{ }^{\prime}-\mathrm{CH}_{3}\right), 6.35(\mathrm{~d}, J=16.1 \mathrm{~Hz}, 1 \mathrm{H}, 1$ '”-H), $7.03\left(\mathrm{~d}, J=16.1 \mathrm{~Hz}, 1 \mathrm{H}, 2^{\prime}\right.$-H), 7.31 (d, $\left.J=7.8 \mathrm{~Hz}, 2 \mathrm{H}, 3^{\prime}, 5^{\prime}-\mathrm{H}\right), 7.38-7.46$ (m, 2H, 6-H, 8-H), $7.59\left(\mathrm{~d}, J=7.8 \mathrm{~Hz}, 2 \mathrm{H}, 3^{\prime}, 5^{\prime}-\mathrm{H}\right), 7.55(\mathrm{~m}, 1 \mathrm{H}, 7-\mathrm{H}), 8.25(\mathrm{~d}, J=8.7 \mathrm{~Hz}, 1 \mathrm{H}, 5-\mathrm{H})$; ${ }^{13} \mathrm{C}-\mathrm{NMR}: \delta$ $21.4\left(4^{\prime}-\mathrm{CH}_{3}\right), 29.7\left(2 \mathrm{xCH}_{3}\right), 71.2$ (C-3”), 117.1 (C-3), 117.5, 117.7 (C-8, C-1”), 123.3 (C-4a), 124.9, 126.1 (C-6, C-2’'), 129.0, 129.5 (C-2',6', C-3',5'), 130.2 (C-1'), 133.2 (C-5), 140.9 (C4'), 143.6 (C-7), 155.4 (C-8a), 162.7 (C-2), 177.5 (C-4). Anal. Calcd. for $\mathrm{C}_{21} \mathrm{H}_{20} \mathrm{O}_{3}$ (320.39): C, $78.73 ; \mathrm{H}, 6.29 \%$. Found: C, 78.92; H, 6.21\%.

Ethyl (E)-3-[2-(4-methylphenyl)-4-oxo-4H-1-benzopyran-3-yl]acrylate (9f). Method A, reaction period: $60 \mathrm{~min}$. Recrystallization from diisopropyl ether. Yield: $76 \%$. White fluffy leaflets, mp 147-148 ${ }^{\circ} \mathrm{C}$. IR: $v_{\max } 2978,1712(\mathrm{C}=\mathrm{O}$, ester), 1654 (C=O, ketone), $1614(\mathrm{C}=\mathrm{C})$, 1467, 1396 (flavone skeleton), 1297, 1287, 1273 (C-O-C), 1165, $756 \mathrm{~cm}^{-1} ;{ }^{1} \mathrm{H}$ NMR: $\delta 1.29$ (t, $J$ $\left.=7.0 \mathrm{~Hz}, 3 \mathrm{H}, \mathbf{C H}_{3} \mathrm{CH}_{2}\right), 2.46\left(\mathrm{~s}, 3 \mathrm{H}, 4^{\prime}-\mathrm{CH}_{3}\right), 4.18$ (q, $\left.J=7.0 \mathrm{~Hz}, \mathrm{CH}_{3} \mathbf{C H}_{2}\right), 7.34$ (d, $J=8.1 \mathrm{~Hz}$, 2H, 3',5'-H), 7.42-7.52 (m, 4H, 6-H, 8-H, 2'-H, 3'-H) 7.55 (d, J = 8.1 Hz, 2H, 2',6'-H), 7.67 $(\mathrm{m}, 1 \mathrm{H}, 7-\mathrm{H}), 8.27(\mathrm{~d}, J=8.4 \mathrm{~Hz}, 1 \mathrm{H}, 5-\mathrm{H}) ;{ }^{13} \mathrm{C}-\mathrm{NMR}: \delta 14.2\left(\mathbf{C H}_{3} \mathrm{CH}_{2}\right), 21.5\left(4^{\prime}-\mathrm{CH}_{3}\right), 60.2$ $\left(\mathrm{CH}_{3} \mathbf{C H}_{2}\right.$ ), 115.2 (C-3), 117.9 (C-9), 123.0 (C-2”), 123.4 (C-4a), 125.4, 126.2 (C-5, C-6), 129.2 (C-1'), 129.3, 129.8 (C-2',6', C-3',5'), 133.8 (C-7), 136.3 (C-3’), 142.0 (C-4'), 155.2 (C-8a), 167.0, 167.9 (C-2, C-1”), 176.8 (C-4). Anal. Calcd. for $\mathrm{C}_{21} \mathrm{H}_{18} \mathrm{O}_{4}$ (334.37): C, 75.43; H, 5.43\%. Found: C, 75.35; H, 5.54\%.

(E)-4'-Chloro-3-styrylflavone (10a). Method A, reaction period: $540 \mathrm{~min}$. Recrystallization from diisopropyl ether. Yield: $45 \%$. Brownish crystalline powder, mp 180-181 ${ }^{\circ} \mathrm{C}$. IR: $v_{\max } 3062$, 3025, 1644 (C=O), 1614 (C=C), 1595, 1487, 1467, 1406, 1389 (flavone skeleton), 1090 (Ar-Cl), 1016, 835, 756, $700 \mathrm{~cm}^{-1} ;{ }^{1} \mathrm{H}$ NMR: $\delta 6.77(\mathrm{~d}, J=16.0 \mathrm{~Hz}, 1 \mathrm{H}, \beta-\mathrm{H}), 7.21-7.53(\mathrm{~m}, 8 \mathrm{H}, \mathrm{Ar}-\mathrm{H})$, 7.69-7.71 (m, 2H, 2',6'-H), $7.81(\mathrm{~m}, 1 \mathrm{H}, 7-\mathrm{H}), 7.79$ (d, J = $16.0 \mathrm{~Hz}, 1 \mathrm{H}, \alpha-\mathrm{H}), 8.29$ (d, J = 7.2 Hz, 1H, 5-H); ${ }^{13} \mathrm{C}-\mathrm{NMR}: \delta 115.3$ (C-3), 117.7 (C-8), 119.4 (C- $\left.\beta\right), 123.4$ (C-4a), 125.2 (C-6), 126.5 (C-3”,5”), 127.7 (C-4”), 128.6, 128.9 (C-3',5', C-2”,6”), 130.7 (C- $\alpha$ ), 131.1 (C-2',6'), 131.5 (C-1'), 133.5 (C-5), 134.9 (C-7), 136.9, 137.9 (C-4', C-1”), 155.3 (C-8a), 167.8 (C-2), 177.2 (C-4). Anal. Calcd. for $\mathrm{C}_{23} \mathrm{H}_{15} \mathrm{ClO}_{2}$ (358.82): C, 76.99; H, 4.21\%. Found: C, 77.11; H, $4.24 \%$.

Ethyl (E)-3-[2-(4-chlorophenyl)-4-oxo-4H-1-benzopyran-3-yl]acrylate (10f). Method A, reaction period: $40 \mathrm{~min}$. Recrystallization from diisopropyl ether. Yield: $45 \%$. Brownish crystalline powder, $\mathrm{mp} 170-171^{\circ} \mathrm{C}$. IR: $\mathrm{v}_{\max } 2982,1712(\mathrm{C}=\mathrm{O}$, ester), 1653 (C=O, ketone), 1616 (C=C), 1471, 1407, 1389 (flavone skeleton), 1296, 1285, 1272 (C-O-C), 1208, 1173, 1094, 762 $\mathrm{cm}^{-1}$; ${ }^{1} \mathrm{H}$ NMR: $\delta 1.27\left(\mathrm{t}, J=7.0 \mathrm{~Hz}, 3 \mathrm{H}, \mathbf{C H}_{3} \mathrm{CH}_{2}\right), 4.18\left(\mathrm{q}, J=7.0 \mathrm{~Hz}, 2 \mathrm{H}, \mathrm{CH}_{3} \mathbf{C H}_{2}\right), 7.38(\mathrm{~d}, J$ $=15.5 \mathrm{~Hz}, 1 \mathrm{H}, \beta-\mathrm{H}), 7.44-7.55(\mathrm{~m}, 6 \mathrm{H}, \mathrm{Ar}-\mathrm{H}, \alpha-\mathrm{H}), 7.69$ (m, 2H, 2',6'-H), 8.27 (d, J = 8.4 Hz, 1H, 5-H); ${ }^{13} \mathrm{C}-\mathrm{NMR}: \delta 14.2\left(\mathbf{C H}_{3} \mathrm{CH}_{2}\right), 60.3\left(\mathrm{CH}_{3} \mathbf{C H}_{2}\right), 115.6(\mathrm{C}-3), 117.8(\mathrm{C}-8), 123.3(\mathrm{C}-4 \mathrm{a})$, 123.9 (C-2”), 125.6, 126.3 (C-5, C-6), 129.0, 131.1 (C-2',6', C-3',5'), 134.0, 135.4 (C-7, C-3”), 
137.5, 137.8 (C-1', C-4'), 155.1 (C-8a), 165.3, 167.7 (C-2, C-1”), 176.6 (C-4). Anal. Calcd. for $\mathrm{C}_{20} \mathrm{H}_{15} \mathrm{ClO}_{4}$ (354.79): C, 67.71; $\mathrm{H} 4.26 \%$. Found: C, 67.55; H, 4.29\%.

(E)-6-Styrylflavone (15a). Method A, reaction period: $60 \mathrm{~min}$. Purified by column chromatography (eluent: hexane-ethyl acetate $=1: 1, \mathrm{v} / \mathrm{v}$ ). Yield: 87\%. Yellow crystalline powder, mp $186-187^{\circ} \mathrm{C}$. IR: $v_{\max } 3057,3012,1645(\mathrm{C}=\mathrm{O}), 1615(\mathrm{C}=\mathrm{C}), 1596,1568,1497,1452$, 1434, 1364 (flavone skeleton), 1238, 976, 907, 844, 811, 771, $692 \mathrm{~cm}^{-1}$; ${ }^{1} \mathrm{H}$ NMR: $\delta 6.80$ (s, 1H, 3-H), 7.08, 7.16 (AB q, $J=16.2 \mathrm{~Hz}, 2 \mathrm{H}, \alpha-\mathrm{H}, \beta-\mathrm{H}), 7.27-7.36$ (m, 3H, 3', 5'-H, 4'-H), 7.53-7.58 $(\mathrm{m}, 6 \mathrm{H}, \mathrm{Ar}-\mathrm{H}), 7.80$ (d, $J=8.3 \mathrm{~Hz}, 1 \mathrm{H}, 7-\mathrm{H}), 7.88(\mathrm{~m}, 2 \mathrm{H}, 2$ ", $6 "-\mathrm{H}), 8.28(\mathrm{~s}, 1 \mathrm{H}, 5-\mathrm{H}) ;{ }^{13} \mathrm{C}-$ NMR: $\delta 107.4$ (C-3), 118.4 (C-8), 122.8 (C-4'), 123.9 (C-4a), 126.2, 126.6 (C-3',5', C-3”, 5”), 126.5 (C-4”), 127.9 (C- $\alpha$ ), 128.7, 128.9 (C-2',6', C-2”, 6”), 130.1 (C- $\beta$ ), 131.5, 131.6 (C-5, C7), 131.6 (C-1'), 134.6 (C-6), 136.7 (C-1”), 155.4 (C-8a), 163.1 (C-2), 178.2 (C-4). Anal. Calcd. for $\mathrm{C}_{23} \mathrm{H}_{16} \mathrm{O}_{2}$ (324.38): C, 85.16; H, 4.97\%. Found: C, 85.00; H, 4.81\%.

(E)-6-(4-Methylstyryl)flavone (15b). Method A, reaction period: $40 \mathrm{~min}$. Purified by column chromatography (eluent: hexane-ethyl acetate $=1: 1, \mathrm{v} / \mathrm{v}$ ). Yield: $88 \%$. Yellow crystalline powder, mp 205-206 ${ }^{\circ} \mathrm{C}$. IR: $v_{\max } 3060,3027,1638(\mathrm{C}=\mathrm{O}), 1613(\mathrm{C}=\mathrm{C}), 1568,1483,1452,1438$, 1362 (flavone skeleton), 823, $772 \mathrm{~cm}^{-1}$; ${ }^{1} \mathrm{H}$ NMR: $\delta 2.35$ (s, 3H, $\mathrm{CH}_{3}$ ), $6.80(\mathrm{~s}, 1 \mathrm{H}, 3-\mathrm{H}) ; 7.04-$ 7.17 (m, 4H, $\alpha-\mathrm{H}, \beta-\mathrm{H}, 3 ", 5 "-\mathrm{H}), 7.39$ (d, J = 8.4 Hz, 2H, 2",6”-H), 7.41-7.52 (m, 4H, 8-H, 3',5'-H, 4'-H), 7.78 (d, J = 8.4 Hz, 1H, 7-H), 7.88 (d, J = 7.7 Hz, 2H, 2',6'-H), 8.27 (s, 1H, 5-H); ${ }^{13} \mathrm{C}-\mathrm{NMR}: \delta 21.2\left(\mathrm{CH}_{3}\right), 107.4(\mathrm{C}-3), 118.3(\mathrm{C}-8), 122.6(\mathrm{C}-\alpha), 123.9(\mathrm{C}-4 \mathrm{a}), 125.6\left(\mathrm{C}-4{ }^{\prime}\right)$, 126.2, 126.5 (C-3',5', C-3”, 5”), 128.9, 129.4 (C-2',6', C-2”,6”), 130.1 (C- $\beta$ ), 131.5, 131.6 (C-5, C-7), 131.7 (C-1'), 134.0, 134.9 (C-6, C-1”), 138.0 (C-4”), 155.4 (C-8a), 163.1 (C-2), 178.2 (C4). Anal. Calcd. for $\mathrm{C}_{24} \mathrm{H}_{18} \mathrm{O}_{2}$ (324.38): C, 85.18; H, 5.36\%. Found: C, 85.01; H, 5.25\%.

(E)-6-(4-Methoxystyryl)flavone (15c). Method A, reaction period: $30 \mathrm{~min}$. Purified by column chromatography (eluent: hexane-ethyl acetate $=1: 1$, v/v). Yield: 41\%. Yellow crystalline powder, mp 198-200 ${ }^{\circ} \mathrm{C}$. IR: $v_{\max } 3063,2840\left(\mathrm{CH}_{3} \mathrm{O}\right), 1651(\mathrm{C}=\mathrm{O}), 1614(\mathrm{C}=\mathrm{C}), 1603,1513$, 1454, 1358 (flavone skeleton), 1304, 1269, 1255 (C-O-C), 1174, 1026 (C-O-C), $824 \mathrm{~cm}^{-1} ;{ }^{1} \mathrm{H}$ NMR: $\delta 3.81\left(\mathrm{~s}, 3 \mathrm{H}, \mathrm{CH}_{3} \mathrm{O}\right), 6.80(\mathrm{~s}, 1 \mathrm{H}, 3-\mathrm{H}), 6.88(\mathrm{~d}, J=8.4 \mathrm{~Hz}, 2 \mathrm{H}, 3$,, $5 "-\mathrm{H}), 6.98(\mathrm{~d}, J=$ $16.2 \mathrm{~Hz}, 1 \mathrm{H}, \beta-\mathrm{H}), 7.14(\mathrm{~d}, J=16.1 \mathrm{~Hz}, 1 \mathrm{H}, \alpha-\mathrm{H}), 7.43-7.52(\mathrm{~m}, 8 \mathrm{H}, \mathrm{Ar}-\mathrm{H}), 7.77(\mathrm{~d}, J=8.4 \mathrm{~Hz}$, 1H, 7-H), 7.89 (m, 2H, 2', 6'-H), $8.25(\mathrm{~s}, 1 \mathrm{H}, 5-\mathrm{H}) ;{ }^{13} \mathrm{C}-\mathrm{NMR}: \delta 55.2\left(\mathrm{CH}_{3} \mathrm{O}\right), 107.3(\mathrm{C}-3)$, 114.1 (C-3",5”), 118.3 (C-8), 122.3 (C- $\alpha$ ), 123.9 (C-4a), 124.5 (C-4'), 126.2 (C-3',5'), 127.8, 128.9 (C-2',6', C-2”,6”), 129.5 (C-1”), 129.7 (C-ß), 131.5 (C-5, C-7), 131.6 (C-1'), 135.0 (C-6), 155.2 (C-8a), 159.5 (C-4”), 163.1 (C-2), 178.3 (C-4). Anal. Calcd. for $\mathrm{C}_{24} \mathrm{H}_{18} \mathrm{O}_{3}$ (354.41): C, 81.34; H, 5.12\%. Found: C, 81.19; H, 5.01\%.

(E)-6-(4-Chlorostyryl)flavone $(\mathbf{1 5 d})$. Method A, reaction period: $30 \mathrm{~min}$. Purified by column chromatography (eluent: hexane-ethyl acetate $=1: 1, \mathrm{v} / \mathrm{v}$ ). Yield: $87 \%$. Pale yellow crystalline powder, mp 202-203 ${ }^{\circ} \mathrm{C}$. IR: $v_{\max }$ 3057, $1640(\mathrm{C}=\mathrm{O}), 1613(\mathrm{C}=\mathrm{C}), 1567,1492,1452,1362$ (flavone skeleton), 1092, 826, $772 \mathrm{~cm}^{-1} ;{ }^{1} \mathrm{H}$ NMR: $\delta 6.80$ (s, 1H, 3-H), 7.04, 7.09 (AB q, $J=16.8$ $\mathrm{Hz}, 2 \mathrm{H}, \alpha-\mathrm{H}, \beta-\mathrm{H}), 7.30$ (d, J = 8.4 Hz, 2H, 3", 5'-H), 7.40 (d, J = 8.4 Hz, 2H, 2", 6"'-H), 7.52 (m, 4H, 8-H, 3' ,5'-H, 4'-H), 7.77 (d, J=8.4 Hz, 1H, 7-H), 7.88 (d, J = 7.0 Hz, 2H, 2',6'-H), 8.26 (s, 1H, 5-H); ${ }^{13} \mathrm{C}-\mathrm{NMR}$ : $\delta 107.4$ (C-3), 118.5 (C-8), 122.9 (C- $\alpha$ ), $124.0(\mathrm{C}-4 \mathrm{a}), 126.2$ (C-3',5'), 
127.2 (C-4'), 127.7 (C-3",5”), 128.8 (C- $\beta$ ), 128.9, 129.0 (C-2',6', C-2”,6”), 131.6, 131.7 (C-5, C-7), 133.5, 134.3, 135.2 (C-6, C-1', C-1"), 155.6 (C-8a), 163.2 (C-2), 178.1 (C-4). Anal. Calcd. for $\mathrm{C}_{23} \mathrm{H}_{15} \mathrm{ClO}_{2}$ (358.82): C, 76.99; H, 4.21\%. Found: C, 77.17; H, 4.13\%.

(E)-6-(3-Hidroxy-3-methylbut-1-enyl)flavone (15e). Method A, reaction period: $30 \mathrm{~min}$. Purified by column chromatography (eluent: hexane-ethyl acetate $=1: 1, \mathrm{v} / \mathrm{v}$ ). Yield: $87 \%$. White crystalline powder, mp $115-117^{\circ} \mathrm{C}$. IR: $v_{\max } 3443(\mathrm{OH}), 3059,2976,1643(\mathrm{C}=\mathrm{O}), 1613(\mathrm{C}=\mathrm{C})$, 1570, 1496, 1486, 1450, 1435, 1362 (flavone skeleton), 1237 (C-OH), 1150, 969, 901, 892, 822, $772 \mathrm{~cm}^{-1} ;{ }^{1} \mathrm{H}$ NMR: $\delta 1.45$ (s, 6H, 2xCH 3 ), 2.05 (s, 1H, OH), 6.45 (d, $J=16.1 \mathrm{~Hz}, 1 \mathrm{H}, 2$ '-H), 6.65 (d, $J=16.1 \mathrm{~Hz}, 1 \mathrm{H}, 1$ '-H), 6.79 (s, 1H, 3-H), 7.47 (m, 4H, 8-H, 3', 5'-H, 4'-H), 7.67 (d, $J=$ $8.4 \mathrm{~Hz}, 1 \mathrm{H}, 7-\mathrm{H}), 7.88\left(\mathrm{~d}, J=7.7 \mathrm{~Hz}, 2 \mathrm{H}, 2^{\prime}, 6{ }^{\prime}-\mathrm{H}\right), 8.17(\mathrm{~s}, 1 \mathrm{H}, 5-\mathrm{H}) ;{ }^{13} \mathrm{C}-\mathrm{NMR}: \delta 29.8$ (2xCH$\left.{ }_{3}\right), 70.9$ (C-3”), 107.3 (C-3), 118.2 (C-8), 122.7 (C-1”), 123.8 (C-4a), 124.6 (C-4'), 126.1 (C-3',5'), 128.9 (C-2',6'), 131.5, 131.8 (C-7, C-2”), 131.6 (C-1), 134.4 (C-6), 139.3 (C-5), 155.3 (C-8a), 163.2 (C-2), 178.3 (C-4). Anal. Calcd. for $\mathrm{C}_{20} \mathrm{H}_{18} \mathrm{O}_{3}$ (306.36): C, 78.41; H, 5.92\%. Found: C, 78.52; H, 6.03\%.

Ethyl (E)-6-(2-phenyl-4-oxo-4H-1-benzopyran-3-yl)acrylate (15f). Method B, reaction period: 300 min. Recrystallization from hexane - abs. ethanol. Yield: 32\%. Brownish crystalline powder, mp 145-146 ${ }^{\circ} \mathrm{C}$. IR: $v_{\max } 3079,2978,1711(\mathrm{C}=\mathrm{O}$, ester), $1636(\mathrm{C}=\mathrm{O}$, ketone), 1607 $(\mathrm{C}=\mathrm{C}), 1568,1496,1483,1453,1358$ (flavone skeleton), 1306, 1238, 1219 (C-O-C), 1197, 1176, 1137, 1032, 1024 (C-O-C), 987 (HC=CH, trans), 908, 827, $772 \mathrm{~cm}^{-1} ;{ }^{1} \mathrm{H}$ NMR: $\delta 1.33$ (t, $J=7.0$ $\left.\mathrm{Hz}, 3 \mathrm{H}, \mathbf{C H}_{3} \mathrm{CH}_{2}\right), 4.25\left(\mathrm{q}, J=7.0 \mathrm{~Hz}, 2 \mathrm{H}, \mathrm{CH}_{3} \mathbf{C H}_{2}\right), 6.49(\mathrm{~d}, J=16.1 \mathrm{~Hz}, 1 \mathrm{H}, 2-\mathrm{H}), 6.54(\mathrm{~s}$, 1H, 3'-H), 7.52-7.58 (m, 4H, 8'-H, 3",5"-H, 4"'- H), 7.71 (d, $J=16.1 \mathrm{~Hz}, 1 \mathrm{H}, 3-\mathrm{H}), 7.81$ (dd, $J=$ $8.4 \mathrm{~Hz}, 2.1 \mathrm{~Hz}, 1 \mathrm{H}, 7-\mathrm{H}), 7.89$ (dd, $J=8.4 \mathrm{~Hz}, 1.8 \mathrm{~Hz}, 2 \mathrm{H}, 2$ ", 6"-H), 8.34 (s, $\left.1 \mathrm{H}, 5^{\prime}-\mathrm{H}\right){ }^{13} \mathrm{C}-$ NMR: $\delta$ 14.2 $\mathbf{C H}_{3} \mathrm{CH}_{2}$ ), 60. $\left.\mathrm{CH}_{3} \mathbf{C H}_{2}\right), 107.6$ (C-3'), 118.8, 119.7 (C-2, C-8'), 124.0 (C-4a'), 125.5 (C-4"), 126.2 (C-3",5”), 129.0 (C-2",6”), 131.4, 131.7 (C-1', C-1"), 131.8, 132.6 (C-3, C7'), 142.4 (C-5'), 156.8 (C-8a'), 163.4 (C-2'), 166.4 (C-1), 177.7 (C-4'). Anal. Calcd. for $\mathrm{C}_{20} \mathrm{H}_{16} \mathrm{O}_{4}$ (320.34): C, 74.99; H, 5.03\%. Found: C, 75.21; H, 5.03\%.

(E)-7-Styrylflavone (16a). Method A, reaction period: $60 \mathrm{~min}$. Recrystallization from hexane abs. ethanol. Yield: $65 \%$. Brownish crystalline powder, mp 153-154 ${ }^{\circ} \mathrm{C}$. IR: $v_{\max } 3058,3020$, $1654(\mathrm{C}=\mathrm{O}), 1618(\mathrm{C}=\mathrm{C}), 1606,1448,1428,1367,1358$ (flavone skeleton), $964(\mathrm{CH}=\mathrm{CH}$, trans), $770 \mathrm{~cm}^{-1}$; ${ }^{1} \mathrm{H}$ NMR: $\delta 6.79(\mathrm{~s}, 1 \mathrm{H}, 3-\mathrm{H}), 7.12(\mathrm{~d}, J=16.3 \mathrm{~Hz}, 1 \mathrm{H}, \beta-\mathrm{H}) ; 7.30(\mathrm{~d}, J=16.3$ $\mathrm{Hz}, 1 \mathrm{H}, \alpha-\mathrm{H}), 7.31-7.41$ (m, 3H, 3",5"-H, 4"-H), 7.53-7.61 (m, 7H, Ar-H), 7.90 (d, J = 7.7 Hz, 2H, 2',6'-H), 8.17 (d, $J=8.4 \mathrm{~Hz}, 1 \mathrm{H}, 5-\mathrm{H}) ;{ }^{13} \mathrm{C}-\mathrm{NMR}: \delta 107.6(\mathrm{C}-3), 115.3(\mathrm{C}-8), 122.8$ (C-4a), 123.2 (C-4'), 125.9 (C- $\alpha$ ), 126.2, 126.8 (C-3',5', C-3",5”), 126.7 (C-6), 128.5 (C-4"), 128.8, 128.9 (C-2',6', C-2",6"), 131.5 (C- $\beta$ ), 131.7 (C-1'), 132.4 (C-5), 136.3 (C-1"), 143.1 (C-7), 156.6 (C-8a), 163.2 (C-2), 177.9 (C-4). Anal. Calcd. for $\mathrm{C}_{23} \mathrm{H}_{16} \mathrm{O}_{2}$ (324.38): C, 85.16; H, 4.97\%. Found: C, 85.31; H, 4.80\%.

(E)-7-(4-Methylstyryl)flavone (16b). Method A, reaction period: 60 min. Purified by column chromatography (eluent: hexane-ethyl acetate $=1: 1, \mathrm{v} / \mathrm{v}$ ). Yield: $92 \%$. Yellow crystalline powder, mp 189-190 ${ }^{\circ} \mathrm{C}$. IR: $v_{\max }$ 3056, 3022, $2917(\mathrm{C}-\mathrm{H}), 1636(\mathrm{C}=\mathrm{O}), 1618(\mathrm{C}=\mathrm{C}), 1604$, 1449, 1430, 1371 (flavone skeleton), $960\left(\mathrm{HC}=\mathrm{CH}\right.$, trans), $826 \mathrm{~cm}^{-1}$; ${ }^{1} \mathrm{H}$ NMR: $\delta 2.36$ (s, 3H, 
$\left.\mathrm{CH}_{3}\right), 6.77$ (s, 1H, 3-H), 7.05 (d, J = 16.1 Hz, 1H, $\left.\beta-\mathrm{H}\right), 7.17-7.21$ (m, 3H, $\beta-\mathrm{H}, 3$ 3",5"-H), 7.41 (d, $J=7.7 \mathrm{~Hz}, 2 \mathrm{H}, 2$ '”,6"-H); 7.50-7.56 (m, 5H, 6-H, 8-H, 3',5'-H, 4'-H); 7.89 (d, J = $7.7 \mathrm{~Hz}$, 2H, 2',6'-H), $8.14(\mathrm{~d}, J=8.4 \mathrm{~Hz}, 1 \mathrm{H}, 5-\mathrm{H}) ;{ }^{13} \mathrm{C}-\mathrm{NMR}: \delta 21.3\left(\mathrm{CH}_{3}\right), 107.6(\mathrm{C}-3), 115.1(\mathrm{C}-8)$, 122.7 (C-4a), 123.2 (C-4'), 125.7, 125.8 (C-6, C- $\alpha$ ), 126.2, 126.8 (C-3',5', C-3”,5”), 129.0, 129.5 (C-2',6', C-2”,6”), 131.5, 132.4 (C- $\beta$, C-5), 131.8 (C-1'), 133.5 (C-1”), 138.6 (C-4”), 143.4 (C-7), 156.6 (C-8a), 163.2 (C-2), 177.9 (C-4). Anal. Calcd. for $\mathrm{C}_{24} \mathrm{H}_{18} \mathrm{O}_{2}$ (324.38): C, 85.18 ; H, 5.36\%. Found: C, 85.12; H, 5.48\%.

(E)-7-(4-Methoxystyryl)flavone (16c). Method A, reaction period: $40 \mathrm{~min}$. Purified by column chromatography (eluent: hexane-ethyl acetate $=1: 1, \mathrm{v} / \mathrm{v}$ ). Yield: 89\%. Yellow crystalline powder, mp 184-185 ${ }^{\circ} \mathrm{C}$. IR: $v_{\max } 3063,3030,2837\left(\mathrm{CH}_{3} \mathrm{O}\right), 1642(\mathrm{C}=\mathrm{O}), 1620(\mathrm{C}=\mathrm{C}), 1599$, 1574, 1509, 1452, 1431, 1417, 1373 (flavone skeleton), 1302, 1251 (C-O-C), 1172, 1030 (C-OC), $960\left(\mathrm{HC}=\mathrm{CH}\right.$, trans), $824 \mathrm{~cm}^{-1} ;{ }^{1} \mathrm{H}$ NMR: $\delta 3.83\left(\mathrm{~s}, 3 \mathrm{H}, \mathrm{CH}_{3} \mathrm{O}\right), 6.77(\mathrm{~s}, 1 \mathrm{H}, 3-\mathrm{H}), 6.90(\mathrm{~d}, J$ $=8.4 \mathrm{~Hz}, 2 \mathrm{H}, 3$ ", $5 "-\mathrm{H}) ; 6.96(\mathrm{~d}, J=16.1 \mathrm{~Hz}, 1 \mathrm{H}, \alpha-\mathrm{H}), 7.19(\mathrm{~d}, J=16.1 \mathrm{~Hz}, 1 \mathrm{H}, \beta-\mathrm{H}), 7.46-$ $7.55(\mathrm{~m}, 7 \mathrm{H}, \mathrm{Ar}-\mathrm{H}), 7.89$ (m, 2H, 2',6'-H), $8.14(\mathrm{~d}, J=7.7 \mathrm{~Hz}, 1 \mathrm{H}, 5-\mathrm{H}) ;{ }^{13} \mathrm{C}-\mathrm{NMR}: \delta 55.3$ $\left(\mathrm{CH}_{3} \mathrm{O}\right), 107.6$ (C-3), 114.2 (C-3”,5”), 114.9 (C-8), 122.5 (C-4a), 123.0 (C- $\left.\alpha\right), 124.5,125.8$ (C-6, C-4'), 126.1 (C-3',5'), 128.2, 128.9 (C-2',6', C-2”,6”), 129.0 (C-1”), 131.5, 132.0 (C-5, C- $\beta$ ), 131.8 (C-1'), 143.5 (C-7), 156.6 (C-8a), 160.0 (C-4”), 163.1 (C-2), 177.9 (C-4). Anal. Calcd. for $\mathrm{C}_{24} \mathrm{H}_{18} \mathrm{O}_{3}$ (354.41): C, 81.34; H, 5.12\%. Found: C, 81.23; H, $4.98 \%$.

(E)-7-(4-Chlorostyryl)flavone $(\mathbf{1 6 d})$. Method A, reaction period: $90 \mathrm{~min}$. Purified by column chromatography (eluent: hexane-ethyl acetate $=1: 1$, v/v). Yield: $68 \%$. Yellow crystalline powder, mp 209-210 ${ }^{\circ} \mathrm{C}$. IR: $v_{\max } 3053,3026,1624(\mathrm{C}=\mathrm{O}+\mathrm{C}=\mathrm{C}), 1559,1486,1451,1436,1374$ (flavone skeleton), 1243, 1082, $954(\mathrm{HC}=\mathrm{CH}$, trans $), 910,817,810,768 \mathrm{~cm}^{-1} ;{ }^{1} \mathrm{H}$ NMR: $\delta 6.78$ (s, 1H, 3-H), 7.08, 7.20 (AB q, $J=16.0 \mathrm{~Hz}, 2 \mathrm{H}, \alpha-\mathrm{H}, \beta-\mathrm{H}), 7.33$ (d, $J=8.1 \mathrm{~Hz}, 2 \mathrm{H}, 3$ ", 5"-H), 7.43 (d, $J=8.1 \mathrm{~Hz}, 2 \mathrm{H}, 2$ ', 6"'-H), 7.51-7.56 (m, 4H, 6-H, 3',5'-H, 4'-H), 7.57 (s, 1H, 8-H), 7.88 (m, 2H, 2',6'-H), 8.15 (d, J = 7.9 Hz, 1H, 5-H); ${ }^{13} \mathrm{C}-\mathrm{NMR}: \delta 107.7$ (C-6), 115.4 (C-8), 123.0 (C4a), 123.2 (C- $\alpha$ ), 125.9 (C-6), 126.1 (C-3”, 5’), 127.2 (C-4'), 128.0 (C-3',5'), 128.9 (C-2',6', C2",6”), 131.0, 131.5 (C-5, C-ß), 131.7 (C-1'), 134.2, 134.8 (C-1", C-4”), 142.7 (C-7), 156.6 (C8a), 163.3 (C-2); 177.8 (C-4). Anal. Calcd. for $\mathrm{C}_{23} \mathrm{H}_{15} \mathrm{ClO}_{2}$ (358.82): C, 76.99; H, 4.21\%. Found: C, 76.84; H, 4.35\%.

(E)-7-(3-Hidroxy-3-methylbut-1-enyl)flavone (16e). Method A, reaction period: 40 min. Purified by column chromatography (eluent: hexane-ethyl acetate $=1: 1, \mathrm{v} / \mathrm{v}$ ). Yield: $76 \%$. White crystalline powder, $\mathrm{mp} 175-176^{\circ} \mathrm{C}$. IR: $v_{\max } 3385(\mathrm{OH}), 3057,3003,1628(\mathrm{C}=\mathrm{O}+\mathrm{C}=\mathrm{C}), 1591$, 1573, 1450, 1376 (flavone skeleton), $1232(\mathrm{C}-\mathrm{OH}), 969\left(\mathrm{CH}=\mathrm{CH}\right.$, trans), 909, $770 \mathrm{~cm}^{-1} ;{ }^{1} \mathrm{H}$ NMR: $\delta 1.47\left(\mathrm{~s}, 6 \mathrm{H}, 2 \mathrm{xCH}_{3}\right), 2.20(\mathrm{~s}, 1 \mathrm{H}, \mathrm{OH}), 6.53,6.66(\mathrm{AB} \mathrm{q}, J=16.1 \mathrm{~Hz}, 2 \mathrm{H}, \alpha-\mathrm{H}, \beta-\mathrm{H})$, 6.78 (s, 1H, 3-H), 7.38 (d, $J=8.4$ Hz, 1H, 6-H), 7.46 (s, 1H, 8-H), 7.50-7.51 (m, 3H, 3', 5' -H, 4' H), $7.88\left(\mathrm{~d}, J=7.7 \mathrm{~Hz}, 2 \mathrm{H}, 2^{\prime}, 6^{\prime}-\mathrm{H}\right), 8.11(\mathrm{~d}, J=8.4 \mathrm{~Hz}, 1 \mathrm{H}, 5-\mathrm{H}) ;{ }^{13} \mathrm{C}-\mathrm{NMR}: \delta 29.7\left(2 \mathrm{xCH}_{3}\right)$, 70.9 (C-3”), 107.5 (C-3), 115.3 (C-8), 122.6 (C-4a), 123.2 (C-1”), 124.8, 125.7 (C-6, C-4'), 126.1 (C-3',5'), 128.9 (C-2',6’), 131.5 (C-2’), 131.6 (C-1'), 141.7 (C-5), 143.0 (C-7), 156.5 (C8a), 163.2 (C-2), 178.1 (C-4). Anal. Calcd. for $\mathrm{C}_{20} \mathrm{H}_{18} \mathrm{O}_{3}$ (306.36): C, 78.41; H, 5.92\%. Found: C, 78.39; H, 6.07\%. 
Ethyl (E)-7-(2-phenyl-4-oxo-4H-1-benzopyran-3-yl)acrylate (16f). Method B, reaction period: 150 min. Recrystallization from hexane - abs. ethanol. Yield: 84\%. Brownish crystalline powder, mp 167-168 ${ }^{\circ} \mathrm{C}$. IR: $v_{\max } 3059,2980,1712(\mathrm{C}=\mathrm{O}$, ester), 1654 (C=O, ketone), 1620 $(\mathrm{C}=\mathrm{C}), 1606,1559,1449,1369$ (flavone skeleton), 1287, 1245, 1185, 1145, 1035, $998(\mathrm{HC}=\mathrm{CH}$, trans), 772, $686 \mathrm{~cm}^{-1}$; ${ }^{1} \mathrm{H}$ NMR: $\delta 1.34\left(\mathrm{t}, J=7.0 \mathrm{~Hz}, 3 \mathrm{H}, \mathbf{C H}_{3} \mathrm{CH}_{2}\right), 4.27(\mathrm{q}, J=7.0 \mathrm{~Hz}, 2 \mathrm{H}$, $\mathrm{CH}_{3} \mathbf{C H}_{2}$ ), 6.60 (d, J = 16.1 Hz, 1H, 2-H), 6.83 (s, 1H, 3'-H), 7.53-7.58 (m, 4H, 6'-H, 3', 5'-H, 4"-H), 7.68 (s, 1H, 8'-H), 7.74 (d, J=16.1 Hz, 1H, 3-H), 7.92 (d, J = 7.7 Hz, 2H, 2",6"-H), 8.22 $\left(\mathrm{d}, J=8.4 \mathrm{~Hz}, 1 \mathrm{H}, 5^{\prime}-\mathrm{H}\right){ }^{13} \mathrm{C}-\mathrm{NMR}: \delta 14.3\left(\mathbf{C H}_{3} \mathrm{CH}_{2}\right), 60.9\left(\mathrm{CH}_{3} \mathbf{C H}_{2}\right), 107.8\left(\mathrm{C}-3{ }^{\prime}\right), 117.7(\mathrm{C}-$ 8'), 121.9 (C-2), 124.1 (C-4”), 124.6 (C-4a'), 126.3 (C-3”,5”), 126.3 (C-6'), 129.1 (C-2”,6”), 131.5 (C-1”), 131.7 (C-3), 139.8 (C-7'), 142.2 (C-5'), 156.3 (C-8a), 163.6 (C-2'), 166.2 (C-1), 177.7 (C-4). Anal. Calcd. for $\mathrm{C}_{20} \mathrm{H}_{16} \mathrm{O}_{4}$ (320.34): C, 74.99; H, 5.03\%. Found: C, 75.05; H, $4.89 \%$.

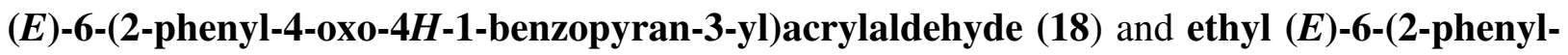
4-oxo-4H-1-benzopyran-3-yl)propionate (19). 6-Bromoflavone (13) (376 mg, $1.249 \mathrm{mmol}$ ) was reacted with acrolein diethyl acetal $(\mathbf{1 7})(0.95 \mathrm{~mL}, 811 \mathrm{mg}, 6.232 \mathrm{mmol})$ under Conditions A at $110{ }^{\circ} \mathrm{C}$. Although the reaction was not complete after 4 hours it was interrupted due to the increase of decomposition and worked-up as usual. The crude product was purified by column chromatography (eluent: hexane-ethyl acetate $=1: 1, \mathrm{v} / \mathrm{v}$ ) to give $146 \mathrm{mg}$ of unreacted starting material 13, $146 \mathrm{mg}$ (59\%) of ester 19 and $36 \mathrm{mg}$ (17\%) of aldehyde 18 in their elution order. Yields were corrected with the recovered starting material. Compound 18. Mp 180-182 ${ }^{\circ} \mathrm{C}$. IR: $v_{\max } 1678(\mathrm{C}=\mathrm{O}$, aldehyde), $1644(\mathrm{C}=\mathrm{O}$, ketone), $1609(\mathrm{C}=\mathrm{C}), 1452,1356$ (flavone skeleton), 1122, 913, $744 \mathrm{~cm}^{-1}$; ${ }^{1} \mathrm{H}$ NMR: $\delta 6.84(\mathrm{dd}, J=15.8 \mathrm{~Hz}, 7.6 \mathrm{~Hz}, 1 \mathrm{H}, 2-\mathrm{H}), 6.91$ (s, 1H, 3'-H), 7.59-7.64 (m, 5H, 3-H, 8'-H, 3",5”-H, 4"'-H), 7.69 (d, J=9.0 Hz, 1H, 7'-H), 7.98 (m, 2H, 2",6"H), $8.45\left(\mathrm{~s}, 1 \mathrm{H}, 5^{\prime}-\mathrm{H}\right), 9.80$ (d, $\left.J=7.6 \mathrm{~Hz}, 1 \mathrm{H}, 1-\mathrm{H}\right) ;{ }^{13} \mathrm{C}-\mathrm{NMR}: \delta 107.9$ (C-3'), 119.3 (C-8'), 124.2 (C-4a'), 126.2 (C-3”,5”), 126.9 (C-4”), 129.1 (C-2”,6”), 129.6 (C-5'), 131.3 (C-6”, C-1”), 132.0, 132.4 (C-2, C-7'), 150.4 (C-3), 157.5 (C-8a'), 163.7 (C-2'), 177.7 (C-4'), 193.2 (C-1). Anal. Calcd. for $\mathrm{C}_{18} \mathrm{H}_{12} \mathrm{O}_{3}$ (276.29): C, 78.25; H, 4.38\%. Found: C, 78.11; H, 4.50\%. Compound 19. Mp 75-76 ${ }^{\circ} \mathrm{C}$. IR: $v_{\max } 3060,2981,1730$ (C=O, ester), 1650 (C=O, ketone), $1620(\mathrm{C}=\mathrm{C})$, 1571, 1485, 1456, 1380, 1366 (flavone skeleton), 1286 (C-O-C), 1175, 775, $690 \mathrm{~cm}^{-1}$; ${ }^{1} \mathrm{H}$ NMR: $\delta 1.25\left(\mathrm{t}, J=6.8 \mathrm{~Hz}, 3 \mathrm{H}, \mathbf{C H}_{3} \mathrm{CH}_{2}\right), 2.70(\mathrm{t}, J=7.6 \mathrm{~Hz}, 2 \mathrm{H}, 2-\mathrm{H}), 3.07(\mathrm{t}, J=7.6 \mathrm{~Hz}, 2 \mathrm{H}, 3-\mathrm{H})$, 4.13 (q, $J=6.8 \mathrm{~Hz}, 2 \mathrm{H}, \mathrm{CH}_{3} \mathbf{C H}_{2}$ ), 6.82 (s, 1H, 3'-H), 7.50-7.58 (m, 5H, 7'-H, 8'-H, 3", 5'-H, 4"'H), 7.92 (m, 2H, 2",6"-H), $8.06\left(\mathrm{~s}, 1 \mathrm{H}, 5^{\prime}\right.$-H); ${ }^{13} \mathrm{C}-\mathrm{NMR}: \delta 14.2\left(\mathbf{C H}_{3} \mathrm{CH}_{2}\right), 30.3(\mathrm{C}-3), 35.6(\mathrm{C}-$ 2), $60.5\left(\mathrm{CH}_{3} \mathbf{C H}_{2}\right), 107.4$ (C-3'), 118.2 (C-8'), 123.8 (C-4a'), 124.5 (C-4”), 126.2 (C-3”,5”), 129.0 (C-2”,6”), 131.5 (C-5'), 131.8 (C-1”), 134.4 (C-5'), 134.4 (C-7'), 137.9 (C-6'), 154.9 (C8a'), 136.3 (C-2'), 172.5 (C-1), 178.4 (C-4'). Anal. Calcd. for $\mathrm{C}_{20} \mathrm{H}_{18} \mathrm{O}_{4}$ (322.36): C, 74.52; H, $5.63 \%$. Found: C, 74.44; H, 5.51\%. 


\section{Acknowledgements}

Financial support of Hungarian Scientific Research Fund (OTKA NI 61336) and TÁMOP 4.2.1./B-09/1/KONV-2010-0007 project is highly appreciated. The latter project is implemented through the New Hungary Development Plan, co-financed by the European Social Fund and the European Regional Development Fund.

\section{References}

1. (a) Malikov, V. M.; Yuldashev, M. P. Chem. Nat. Compd. 2002, 38, 358. (b) Harborne, J. B.; Baxter, H. The Handbook of Natural Flavonoids, Wiley: Chichester, 1999; Vol. 1. (c) Beecher, G. R. J. Nutr. 2003, 133, 3248S. (d) Havsteen, B. H. Pharmacol. Ther. 2002, 96, 67. (e) Middleton, E.; Kandaswami, C.; Theoharides, T. C. Pharmacol. Rev. 2000, 52, 673. (f) Boccard, J.; Bajot, F.; Di Pietro, A.; Rudaz, S.; Boumendjel, A.; Nicolle, E.; Carrupt, P.-A. Eur. J. Pharm. Science 2009, 36, 254.

2. (a) Nagao, T.; Abe, F.; Kinjo, J.; Okabe, H. Biol. Pharm. Bull. 2002, 250, 875. (b) Daskiewicz, J.-B.; Depeint, F.; Viornery, L.; Bayet, C.; Geraldine, C.-S.; Comte, G.; Gee, J.-M.; Johnson, I.-T.; Ndjoko, K.; Hostettmann, K.; Barron, D. J. Med. Chem. 2005, 48, 2790. (c) Rao, Y. K.; Fang, S.-H.; Tzeng, Y.-M. Bioorg. Med. Chem. 2005, 13, 6850. (d) Kim, Y.-W.; Hackett, J. C.; Brueggemeier, R. W. J. Med. Chem. 2004, 47, 4032. (e) Su, B.; Hackett, J. C.; Diaz-Cruz, E. S.; Kim, Y.-W.; Brueggemeier, R. W. Bioorg. Med. Chem. 2005, 13, 6571. (f) Yang, C. S.; Prabhu, S.; Landau J. Drug Metab. Rev. 2001, 33, 237. (g) Haghiac, M.; Walle, T. Nutr. Cancer 2005, 53, 220. (h) Holder, S.; Lilly, M.; Brown, M. L. Bioorg. Med. Chem. 2007, 15, 6463. (i) Cabrera, M.: Simoens, M.; Falchi, G.; Lavaggi, M. L.; Piro, O. E; Castellano, E. E.; Vidal, A.; Azqueta, A.; Monge, A.; Lopez de Ceráin, A.; Sagrera, G.; Seoane, G.; Ceretto, H.; González, M. Bioorg. Med. Chem. 2007, 15, 3356. (j) Ahn, Y. M.; Vogeti, L.; Liu, C.-J.; Santhapuram, H. K. R.; White, J. M.; Vasandani, V.; Mitscher, L. A.; Lushington, G. H.; Hanson, P. R.; Powell, D. R.; Himes, R. H.; Roby, K. F.; Ye, Q.; Georg, G. I. Bioorg. Med. Chem. 2007, 15, 702. (k) Li-Weber, M. Cancer Treatment Rev. 2009, 35, 57. (1) Brožič, P.; Kocbek, P.; Sova, M.; Kristl, J.; Martens, S.; Adamski, J.; Gobec, S.; Lanišnik Rižner, T. Mol. Cell. Endocrin. 2009, 301, 229.

3. (a) van Acker, F. A. A.; Hageman, J. A.; Haenen, G. R. M. M.; van der Vijgh, W. J. F.; Bast, A.; Menge, W. M. P. B. J. Med. Chem. 2000, 43, 3752. (b) Thana, N. N.; Fotso, S.; Poeggeler, B.; Hardeland, R.; Laatsch, H. Z. Naturforsch. 2006, 61b, 1. (c) van Hoyweghen, L.; Karalic, I.; van Calenbergh, S.; Deforce, D.; Heyerick, A. J. Nat. Prod. 2010, 73, 1573.

4. Kavvadias, D.; Sand, P.; Youdim, K. A.; Qaiser, M. Z.; Rice-Evans, C.; Baur, R.; Sigel, E.; Rausch, W.-D.; Riederer, P.; Schreier, P. British K. Pharmacol 2004, 142, 811.

5. Gao, H.; Kawabata, J. Bioorg. Med. Chem. 2005, 13, 1661.

6. Gao, G.-Y.; Li, D.-J.; Keung, W. M. J. Med. Chem. 2001, 44, 3320.

7. Traxler, P.; Green, J.; Mett, H.; Séquin, U.; Furet, P. J. Med. Chem. 1999, 42, 1018. 
8. Joo, Y. H.; Kim, J. K.; Kang, S.-H.; Noh, M.-S.; Ha, J.-Y.; Choi, J. K.; Lim, K. M.; Lee, C. H.; Chung, S. Bioorg. Med. Chem. 2003, 13, 413.

9. For a recent review see Silva, A. M. S.; Pinto, D. C. G. A.; Cavaleiro, J. A. S.; Lévai, A.; Patonay, T. Arkivoc 2004, (vii), 106.

10. (a) Reddy, B. P.; Krupadanam, G. L. D. J. Heterocycl. Chem. 1996, 33, 1561. (b) Pinto, D. C. G. A.; Silva, A. M. S.; Cavaleiro, J. A. S. J. Heterocycl. Chem. 1996, 33, 1887. (c) Silva, A. M. S.; Cavaleiro, J. A. S.; Elguero, J. Liebigs Ann. Chem. 1997, 2065. (d) Silva, V. M. L.; Silva, A. M. S.; Pinto, D. C. G. A.; Cavaleiro, J. A. S.; Patonay, T. Synlett 2004, 2717. (e) Patonay, T.; Kiss-Szikszai, A.; Silva, V. M. L.; Silva, A. M. S.; Pinto, D. C. G. A.; Cavaleiro, J. A. S.; Jekő, J. Eur. J. Org. Chem. 2008, 1937. (f) Silva, V. M. L.; Silva, A. M. S.; Pinto, D. C. G. A.; Cavaleiro, J. A. S.; Vasas, A.; Patonay, T. Monatsh. Chem./Chem. Monthly 2008, 139, 1307 and the references cited therein.

11. (a) Gomes, A.; Fernandes, E.; Silva, A. M. S.; Santos, C. M. M.; Pinto, D. C. G. A.; Cavaleiro, J. A. S.; Lima, J. L. F. C. Bioorg. Med. Chem. 2007, 15, 6027. (b) Gomes, A.; Freitas, M.; Fernandes, E.; Lima, J. L. F. C. Mini-Reviews Med. Chem. 2010, 10, 1.

12. (a) Goyal, S.; Parthasarathy, M. R. Indian J. Chem. 1992, 31B, 391. (b) Lokshin, V.; Heynderickx, A.; Samat, A.; Pepe, G.; Guglielmetti, R. Tetrahedron Lett. 1999, 40, 6761.

13. Clarke, D. S.; Gabbutt, C. D.; Hepworth, J. D.; Heron, B. M. Tetrahedron Lett. 2005, 46, 5515.

14. (a) Davies, S. G.; Mobbs, B. E. J. Chem. Soc. Perkin Trans 1. 1987, 2597. (b) Dawood, K. M. Tetrahedron 2007, 63, 9642. (c) Klymchenko, A. S.; Mély, Y. Tetrahedron Lett. 2004, 45, 8391. (d) Klymchenko, A. S.; Stoeckel, H.; Takeda, K.; Mély, Y. J. Phys. Chem. B. 2006, 110, 13624. (e) Santos, C. M. M.; Silva, A. M. S.; Cavaleiro, J. A. S. Synlett 2005, 20, 3095. (f) Santos, C. M. M.; Silva, A. M. S.; Cavaleiro, J. A. S. Synlett 2007, 20, 3113. (g) Dahlen, K.; Grotli, M.; Luthman, K. Synlett 2006, 897. (h) Dahlen, K.; Wallén, E. A. A.; Grotli, M.; Luthman, K. J. Org. Chem. 2006, 71, 6863. (j) Tatsuta, K.; Kasai, S.; Amano, Y.; Yamaguchi, T.; Seki, M.; Hosokawa, S. Chem. Lett. 2007, 36, 10 .

15. (a) Patonay, T.; Vasas, A.; Kiss-Szikszai, A.; Silva, A. M. S.; Cavaleiro, J. A. S. Aust. J. Chem. 2010, 63, 1582. (b) Vasas, A.; Patonay, T.; Kónya, K.; Silva, A. M. S.; Cavaleiro, J. A. S. Aust. J. Chem. 2011, 64, 647.

16. Kitanaka, S.; Takido, M. Chem. Pharm. Bull. 1991, 39, 3254.

17. Gutierrez-Lugo, M.-T.; Deschamps, J. J.; Holman, T. R.; Suarez, E.; Timmermann, B. N. Planta Med. 2004, 70, 263.

18. Shimizu, K.; Kondo, R.; Sakai, K.; Lee, S.-H.; Sato, H. Planta Med. 1998, 64, 408.

19. (a) Saraswathy, A.; Balakrishna, K.; Bhima Rao, R.; Allirani, T.; Patra, A.; Pichal, R. Fitoterapia 1998, 69, 463. (b) Sheu, Y.-W.; Chiang, L.-C.; Chen, I.-S.; Chen, Y.-C.; Tsai, I.-L. Planta Med. 2005, 71, 1165. (c) Ali, M. I.; Ahmed, Z.; Wafflo, A. F. K.; Ali, M. S. Nat. Prod. Commun. 2010, 5, 889.

20. (a) Maximo, P.; Lourenco, A.; Feio, S. S.; Roseiro, J. C. Phytochemistry 1988, 27, 3637. (b) Ribeiro, A. B.; Abdelnur, P. V.; Garcia, C. F.; Belini, A.; Severino, V. G. P.; da Silva, M. F. G. F.; Fernandes, J. B.; Vieira, P. C.; Carvalhi, S. A.; Souza, A. A.; Machado, M. A. J. Agric. Food Chem. 2008, 56, 7815. 
21. (a) Subba Rao, N. V.; Veerabhadrarao, J.; Seshadri, T. R. Proc. - Indian Acad. Sci. 1939, 10A, 65. (b) Ranga Rao, R.; Tiwari, A. K.; Reddy, P. P.; Babu, K. S.; Ali, A. Z.; Madhusudana, K.; Rao, J. M. Bioorg. Med. Chem. 2009, 17, 5170.

22. Bognár, R.; Rákosi, M.; Litkei, G. Acta Chim. Acad. Sci. Hung. 1962, 34, 353.

23. Patonay, T.; Cavaleiro, J. A. S.; Lévai, A.; Silva, A. M. S. Heterocycl. Comm. 1997, 3, 223.

24. Diesbach, H.; Kramer, H. Helv. Chim. Acta 1945, 28, 1399.

25. Rho, H. S.; Ko, B.-S.; Kim, H. K.; Ju, H.-S. Synth. Commun. 2002, 32, 1303.

26. Reddy, N. J.; Bokadia, M.; Sharma, T.; Donelly, J. A. J. Org. Chem. 1981, 46, 638.

27. Chen, F. C.; Chang, C. T. J. Chem. Soc. 1958, 146. 